\title{
Terapia combinada para el control de la dependencia a nicotina
}

\author{
DANIEL SEIJAS B.*
}

\section{Combination therapy for nicotine dependence control}

This therapy is a combination of medicines consisting of nicotine replacement therapy (NRT) using a prolonged modality such as the patch, along with a short-acting medicine such as chewing gum, lozenge, gum, or nasal inhaler). This means two or more drugs approved and demonstrated useful for cessation of smoking with or without the support of NRT. It is very important to consider medical and psychiatric comorbidity because the population that persists addicted is increasingly complex in terms of comorbidities and high addictive level. Most of the combination therapies use NRT associated with bupropion or varenicline. There is evidence on the effectiveness and safety of TRN used in both modalitres (long and short acting) in combination with varenicline or bupropion. However, safety evidence is not robust for the combination modality as it is for, each drug as monotherapy, since adverse effects are added so it is suggested to reserve the combinations for people with high level of addiction and / or history of failure in previous attempts with monotherapy. In summary, therapy with demonstrated effectiveness as NRT, bupropion and varenicline can be used in double or triple combination, prefering the use of short acting NRT added to one of the oral drugs to alleviate smoking anxiety.

Key words: Smoking cessation; Tobacco use cessation products; Bupropion; Nicotine; Varenicline.

\section{Resumen}

La terapia combinada es la de mezcla de farmacos para al cesación del tabaquismo, tal como terapias de reemplazo nicotínico (TRN) en modalidad prolongada como es el parche junto a una modalidad de acción corta como puede ser chicle, goma, lozenge, pastillas o inhalador nasal), es decir dos o más fármacos aprobados y demostrados útlies para el cese del tabaco con o sin el apoyo de TRN. Es muy importante considerar la comorbilidad médica y psiquiatrica porque la población que persiste adicta es cada vez más compleja en términos de comorbilidades y elevado nivel adictivo. La mayor parte de las terapias combinadas usan TRN asociadas a bupropión o vareniclina. Existe evidencia sobre efectividad y seguridad de las TRN utilizadas entre ellas o en asociación a vareniclina o bupropión, sin embargo, la evidencia sobre seguridad en la modalidad combinada no es tan robusta como la que existe para cada fármaco en monoterapia, ya que los efectos adversos se suman de manera que se sugiere reservar las combinaciones para personas con alto nivel de adicción y/o con historia de fracaso en intentos previos con monoterapia. En suma, los fármacos de demostrada efectividad y seguridad como TRN, bupropión y vareniclina pueden usarse en combinación doble o triple, preferenciando el uso de TRN de corta acción cuando se adiciona a alguno de los fármacos orales para aliviar la ansiedad por fumar.

Palabras clave: Dejar de fumar; productos para dejar de fumar; nicotina; bupropión; vareniclina.

La terapia combinada se refiere fundamentalmente a la estrategia de combinación de terapias de reemplazo nicotínico (TRN) en que se combina una modalidad prolongada como es el parche a una de acción corta como puede ser chicle, losenge o pastillas, inhalador o spray nasal) o la combinación de dos o más fármacos aprobados y demostrados útiles para el cese del tabaco con o

* Jefe Unidad de Adicciones y Tabaco Clínica Las Condes, Santiago de Chile. Jefe Departamento de Psiquiatría Clínica Las Condes. 
sin el apoyo de TRN. En definitiva, las combinaciones pueden incluir un fármaco oral + TRN de acción larga (parches) y/o corta (chicles) o dos fármacos orales con o sin TRN asociado. En el caso de los fármacos orales como bupropión o vareniclina, el uso de alguna TRN de acción corta cumple el rol de disminuír el antojo o "craving" por fumar, aliviando la ansiedad por fumar y evitando una posible recaida.

Es importante destacar que los pacientes fumadores tienden a presentar con mayor frecuencia que la poblacion general, un sinumero de patologías cardiovasculares, respiratorias, metabólicas y psquiátricas que hacen considerar la combinación de fármacos en uso, tanto por los efectos de potencianción que pudieran presentar, así como los efectos colaterales y reacciones adversas. Así entonces, es importante considerar la comorbilidad médica y psiquiátrica antes de prescribir un tratamiento de cesación sobretodo en los tiempos actuales en que la población que persiste adicta es cada vez más compleja en términos de comorbilidades y elevado nivel adictivo ${ }^{1}$. Es así como en un ensayo randomizado de 127 fumadores $>$ 18 años, la terapia combinada de bupropión más TRN en forma flexible (chicles) logró significativamente mayor abstinencia a las 26 semanas, 35 versus $19 \%$. Si bien hubo mayor insomnio y ansiedad, no hubo diferencia en la frecuencia de descontinuación (65\% para ambos grupos) $)^{1}$.

Actualmente la población incorporada en los estudios es cada vez más adicta como se ilustra en la literatura en que hasta un $58 \%$ de los fumadores continúan fumando luego del diagnóstico de un nuevo cáncer y $50 \%$ de fumadores vuelven a fumar a los seis meses de un infarto. La evidencia sustenta además que fumadores con alto nivel de adicción a nicotina (AN) se benefician de tratamientos de mayor intensidad, incluyendo combinaciones de fármacos, $\mathrm{y}$ duración por sobre las clásicas 8-12 semanas sugeridas en general para las terapias farmacológicas de las $\mathrm{AN}^{2}$.

La mayor parte de las terapias combinadas usan TRN asociadas a alguno de los fármacos más utilizados, bupropión o vareniclina. Ellas han demostrado ampliamente ser seguras y efectivas $^{3,4}$ y si bien en el mundo se encuentran disponibles en variadas formulaciones (chicles, parches, inhaladores, spray nasales y lozenges) en nuestro país hoy, solo es posible encontrarlas en la formulación de chicles de nicotina de 2 y $4 \mathrm{mg}$, gomas de 2 y $4 \mathrm{mg}$ y los parches de 7, 14 y $21 \mathrm{mg}$ estaran disponibles en 2018. Se ha demostrado la efectividad y seguridad de las TRN utilizadas entre ellas o en asociación a vareniclina o bupropión sin embargo, la evidencia de la seguridad en la modalidad combinada no es tan robusta como la que existe para cada fármaco en monoterapia ya que los efectos adversos se suman de manera que se sugiere reservar las combinaciones para personas con alto nivel de AN y/o con historia de fracaso en intentos previos con monoterapia. Generalmente las TRN se inician el mismo día del día " $D$ " y tanto vareniclina y bupropión requieren inicio una semana previa a este día para lograr niveles útiles en este momento ${ }^{5,6}$.

La combinación de bupropión y vareniclina ha sido demostrada efectiva como lo ilustra un ensayo de tratamiento adaptativo aleatorizado, de grupos paralelos, doble ciego, en que se identificaron 222 fumadores que no mostraron una reducción de más del $50 \%$ del consumo de tabaco después de 1 semana de tratamiento con parches de nicotina. Los fumadores fueron asignados aleatoriamente a recibir 12 semanas de vareniclina más bupropión o vareniclina más placebo. La medida de resultado primaria fue la abstinencia de fumar continua en las semanas 8-11 después de la fecha meta de abstinencia ${ }^{7}$.

Ambos tratamientos fueron bien tolerados. Los participantes que recibieron el tratamiento de combinación tuvieron una tasa de abstinencia significativamente más alta que los que recibieron vareniclina más placebo $(39,8 \%$ frente a $25,9 \%$; $\mathrm{OR}=1,89$, IC $95 \%=1,07,3,35)$.

El tratamiento de combinación tuvo un efecto significativamente mayor sobre la tasa de abstinencia en fumadores varones (odds ratio $=$ $4,26$, IC $95 \%=1,73,10,49)$ que en las mujeres fumadoras (odds ratio $=0,94$, IC 95\% $=0,43$, $2,05)$. También tuvo un efecto significativamente mayor en los fumadores altamente dependientes de la nicotina (odds ratio $=3,51$, IC 95\% $=1,64$, $7,51)$ que en los fumadores con niveles más bajos de dependencia (odds ratio $=0,71$ IC 95\% $=$ $0,28,1,80)$.

Entre los fumadores que no mostraron una respuesta inicial suficiente con parches de nicotina, el tratamiento combinado con vareniclina y bupropión demostró ser más eficaz que la vareniclina sola para hombres fumadores y en fumadores con un alto grado de dependencia a la nicotina ${ }^{7}$.

Otro estudio mostró el beneficio de la terapia combinada en pacientes con comorbilidades. Este estudio randomizado de combinación triple para 127 fumadores medicamente enfermos, en atención primaria, comparó el dar parches de nicotina solos o combinados con un inhalador de nicotina y combinación además con bupropión por un período de 10 semanas $^{12}$. El uso de terapia combinada dio mejores resultados que los parches de nicotina solos en atención primaria a los 7 días y 
a las 26 semanas $^{8}$.

Un 35\% de los pacientes en terapia combinada vs $19 \%$ parches solos lograron abstinencia a las 26 semanas. El odds ratio en el grupo combinado fue de 2,57 (CI, 1,05 to 6,32; p < 0.041). El tiempo medio de recaída fue significativamente mas largo en el combinado 65 días vs parches 23 días. Algunos efectos colaterales fueron más frecuentes en el grupo combinado como ansiedad $25 \%$ vs $9 \%$ en el de parches e insomnio mientras que el insomnio se presento en combinación en un $22 \%$ vs $3 \%$ en parches solos 8 .

En general se ha visto que todas las terapias de combinación más que doblan la posibilidad de que los fumadores dejen de fumar comparado con placebo ${ }^{9}$. Cuando se comparó con parches solos, sólo la combinación de $2 \mathrm{mg}$ de Vareniclina más parche de nicotina más chicle o spray tuvieron resultados significativamente superiores al parche solo.

La combinación de parche más chicle, lozenge o spray llegaba a triplicar la posibilidad de abstinencia a largo plazo y se asociaba a mayor posibilidad de abstinencia que el parche solo. Estos efectos fueron comparables al uso de Vareniclina $2 \mathrm{mg} / \mathrm{día}^{9}$.

En suma, los fármacos de demostrada efectividad y seguridad como TRN, bupropión y vareniclina pueden usarse en combinación doble o triple, preferenciando el uso de TRN de corta acción cuando se adiciona a alguno de los fármacos orales para aliviar la ansiedad por fumar. En general está demostrado que los efectos adversos se incrementan al utilizar combinaciones de manera que estas se reservan para los pacientes refractarios al uso de monoterapia, post fracaso en una terapia bien llevada y en pacientes con alto nivel de adicción nicotínica ${ }^{10}$.

\section{Bibliografía}

1.- STEINBERG MB, GREENHAUS S, SCHMELZER AC, BOVER MT, FOULDS, et al. Triple-combi- nation pharmacotherapy for medically ill smokers: a randomized trial. Ann Intern Med 2009 Apr 7; 150(7): 447-54.

2.- A clinical practice guideline for treating tobacco use and dependence: A US Public Health Service report. The Tobacco Use and Dependence Clinical Practice Guideline Panel, Staff and Consortium Representatives. J Am Med Assoc 2000; 283: 3244-54.

3.- FIORE MC, JAÉN CR, BAKER TB, et al. Treating Tobacco Use and Dependence: 2008 Update. Clinical Practice Guideline. Rockville, MD: U.S. Department of Health and Human Services. Public Health Service. May 2008.

4.- STEAD LF, PERERA R, BULLEN C, MANT D, HARTMANN-BOYCE J, CAHILL K, LANCASTER $\mathrm{T}$. Nicotine replacement therapy for smoking cessation. Cochrane Database of Systematic Reviews 2012, nov 14: 11 .

5.- BULLEN C, HOWE C, LIN RB, GRIGG M, LAUGESEN M, MCROBBIE H, et al. Pre-cessation nicotine replacement therapy: Pragmatic randomized trial. Addiction 2010; $105: 1474-83$.

6.- SHIFFMAN S, FERGUSON SG. Nicotine patch therapy prior to quitting smoking: A meta-analysis. Addiction 2008; 103: 557-63.

7.- ROSE JE, BEHM FM. Combination Treatment With Varenicline and Bupropion in an Adaptive Smoking Cessation Paradigm. Am J Psychiatry 2014; 171: 1199205.

8.- GERACI M, BELPARIO P. Recommendations for Use of Combination Therapy in Tobacco Use Cessation. Disponible en: https://www.healthquality.va.gov/guidelines/ $\mathrm{CD} / \mathrm{mtu} /$ tuc_combination_therapy.pdf

9.- VOGELER T, MCCLAIN C, EVOY KE. Am J Drug Alcohol Abuse. 2016 Mar;42(2):129-39. doi: 10.3109/00952990.2015.1117480. Epub 2016 Jan 25.Combination bupropion SR and varenicline for smoking cessation: a systematic review.

10.- Recommendations for Use of Combination Therapy in Tobacco Use Cessation. April 2009. VHAPharmacy Benefits managementservices, Medical advisory Panel, Tobacco Use Cessation Technical Advisory Group, and Public health strategic Healthcare Group. (USPHS CPG). April 2009. 